\title{
Avalanches and Non-Gaussian Fluctuations of the Global Velocity of Imbibition Fronts
}

\author{
Ramon Planet, ${ }^{1}$ Stéphane Santucci, ${ }^{2}$ and Jordi Ortín ${ }^{1}$ \\ ${ }^{1}$ Departament d'Estructura i Constituents de la Matèria, Universitat de Barcelona, Martí i Franquès 1, E-08028 Barcelona, Spain \\ ${ }^{2}$ Department of Physics and Physics of Geological Processes, Oslo University, \\ Post Office Boks 1048 Blindern, N-0316 Oslo 3, Norway \\ (Received 16 September 2008; published 3 March 2009)
}

\begin{abstract}
We present an experimental study of the global velocity $V(t)$ of a viscous fluid interface during forcedflow imbibition in a disordered medium. Our high resolution setup shows that the fronts display an intermittent behavior signature of a burstlike dynamics, with power-law distributed avalanches. When measured at scales comparable to the correlation length, velocity fluctuations follow an asymmetric nonGaussian distribution, whose skewness increases with decreasing measuring window and/or injection flow rate, offering the effective number of degrees of freedom probed in our experiment.
\end{abstract}

PACS numbers: 47.56.+r, 05.40.- a, 47.53.+n, 68.35.Ct

The pioneering work of BHP [1] -on non-Gaussian fluctuations of the power consumption in a turbulent reactor, and of the magnetization of an $X Y$ Ising modeltriggered an upsurge of studies concerning the fluctuations of global quantities in correlated systems [1-6]. When the correlation length is of the order of the system size, it has been proposed that the PDF of a global quantity is asymmetric and can be approximated very well by a generalized Gumbel distribution (GG) [1-3]. Most of these studies are purely theoretical, and experimental results are much more scarce $[1,5]$. Experimental evidence of a GG-like distribution has been reported very recently for the fluctuations of the spatially averaged order parameter close to the critical point of a second-order phase transition [6]. It appears of crucial importance to bring new experimental evidences and a detailed characterization of such non-Gaussian fluctuations, in particular, for nonequilibrium systems. In the present work this kind of evidences are provided for interfacial fluctuations in fluid invasion of disordered media, a nonequilibrium system that spontaneously develops longrange correlations.

We present here an experimental study of the spatially averaged velocity $V(t)$ of an air-liquid interface during forced-flow imbibition of a viscous wetting liquid by a disordered medium. While the scaling properties of the interfacial morphology in imbibition experiments have been studied extensively (see the review [7] and references therein), the dynamics of the process has not received the same attention [8]. Using a high resolution fast camera, we follow the imbibition front and observe a complex intermittent dynamics, due to the interplay between competing forces at different time and length scales. Indeed, viscous pressure and interfacial tension tend to make the interface stable on large length scales, while variations in gap spacing in the disordered medium give rise to capillary pressure fluctuations that destabilize it on short length scales. As a result, the front undergoes a kinetic roughening process that leads to a statistically stationary state with critical interfacial fluctuations $[7,9]$.
First, we show that the large velocity fluctuations correspond to avalanches that follow power-law distributions for a wide range of length and duration scales. Then, we consistently find a scaling behavior between sizes and durations of those avalanches, $S \sim T^{x}$, with $x \simeq 1.31$ in quantitative agreement with numerical results [10]. The second part of the Letter focuses on the fluctuations of the mean velocity $V(t)$. Our results show that the PDF of the fluctuations of $V$ is very well approximated by a GG distribution when the size of the measuring window is comparable to the lateral correlation length. The asymmetry of the PDF increases as the measuring window and/or the flow rate are reduced. Our experiments therefore provide the first experimental evidence of GG-like fluctuations of a global magnitude in a critical nonequilibrium system that spontaneously develops long-range correlations.

Experimental setup.-Our model disordered medium is a horizontal Hele-Shaw cell, made of two parallel glass plates of size $190 \times 500 \mathrm{~mm}^{2}$ separated by a narrow gap that takes two different values, 0.40 and $0.46 \mathrm{~mm}$, randomly distributed in space (provided by a random distribution of copper square patches $l_{d}^{2}=0.40 \times 0.40 \mathrm{~mm}^{2}$ on a fiberglass board attached to the bottom plate, covering $35 \%$ of the total area) [11]. We prewet the cell with a very thin oil layer to minimize the possible influence of precursor films and/or wetting heterogeneities. A syringe pump drives the viscous liquid-a silicone oil (Rhodorsil $47 \mathrm{~V})$ of kinematic viscosity $\nu=50 \mathrm{~mm}^{2} / \mathrm{s}$, density $\rho=998 \mathrm{~kg} / \mathrm{m}^{3}$, and oil-air surface tension $\sigma_{\mathrm{oa}}=$ $20.7 \mathrm{mN} / \mathrm{m}$ at room temperature - at constant flow rate. The oil wets perfectly (zero contact angle) all the surfaces in contact. The range of front velocities $v$ explored here $(0.05-0.25 \mathrm{~mm} / \mathrm{s})$ corresponds to small capillary numbers $\mathrm{Ca}=\mu v / \sigma_{\mathrm{oa}}<10^{-3}$, ensuring that front roughening in our experiments is due to capillary disorder, not to permeability disorder.

The imbibition fronts are monitored within a measurement window (ROI) of lateral size $L=125 \mathrm{~mm}$ (smaller 
than the whole width of the cell, $190 \mathrm{~mm}$ ), centered with the cell, to avoid any distortion of the interfaces produced by the side walls. We use a Motion Pro X3 plus videocamera with $1280 \times 276$ pixels. The spatial resolution is thus $r=0.098 \mathrm{~mm} /$ pixel, more than $4 \times 4$ pixels per square patch. In each experiment an initially flat front propagates for about $150 \mathrm{~mm}$ in the $y$ direction of the cell. In the course of this process the interface reaches a statistically stationary state (with a saturated width). We follow the front propagation in the next $100 \mathrm{~s}$, recording 10000 images of the ROI ( $\delta t=0.01 \mathrm{~s}$ between consecutive images). The large temporal resolution of our measurements ensures that the front does not move forward by more than one pixel in one time step. The front position $h(x, t)$ is extracted using a method of edge detection. The waiting time $\mathrm{wt}(x, y)$ at the front position $(x, y=h(x, t))$ is computed as the amount of time (number of counts $\times \delta t$ ) during which the imbibition front was pinned at this precise position. From $\mathrm{wt}(x, y)$ we measure the local velocity as $v(x, h(x, t))=r / w t(x, h(x, t))$. This method was recently developed to study the local waiting time fluctuations of a crack front [12]. The uncertainty of $\pm \delta t$ on the value of wt can be significant when wt is small. However, small wt $(\leq 10 \delta t)$ are very seldom $(<0.2 \%$ of the measurements), thanks to our relatively high acquisition rate. Finally the global velocity of the front in a window of size $l \leq L$, is obtained from the spatial average $V_{l}(t) \equiv(1 / l) \times$ $\int_{0}^{l} v(x, t) d x$. In the sequel we drop the subindex $l$ to make the notation simpler. In this Letter, we focus on the statistics of the global velocity $V(t)$.

The behavior of $V(t)$ for a window $l=L$ and different injection rates is shown in Fig. 1. As expected, the range of variation of $V(t)$ depends on the injection rate. For all the experiments performed, while the injection rate is constant, $V(t)$ is a jerky signal with a complex intermittent behavior characterized by large fluctuations. We have verified that these fluctuations do not originate from the injection system or the digitization procedure, by performing the same experiments in a smooth cell. Even though the forced-flow condition enforces mass conservation in three dimensions, fluctuations of the gap thickness between two prescribed values gives rise to fluctuations of the global velocity of the projected one-dimensional interface. Moreover, the global velocity of a fraction of size $l$ of the interface displays even larger fluctuations, due to the finite system size. It is remarkable that these time series look very similar to the ones obtained recently by phase-field numerical simulations [10].

Avalanche dynamics.-We consider as avalanches the occurrences of $V(t)$ above an arbitrary threshold. Let $S$ and $T$, respectively, be the size and duration of an avalanche, as shown in the top panel of Fig. 1. $S$ is the extra displacement of the average front in the avalanche. Both definitions depend on an arbitrary threshold $V_{c}=\langle V\rangle+c \sigma$, where $\langle V\rangle$ is the time average and $\sigma^{2}$ the variance of $V(t)$ over the total duration of an experiment, and $c$ is an arbitrary clip

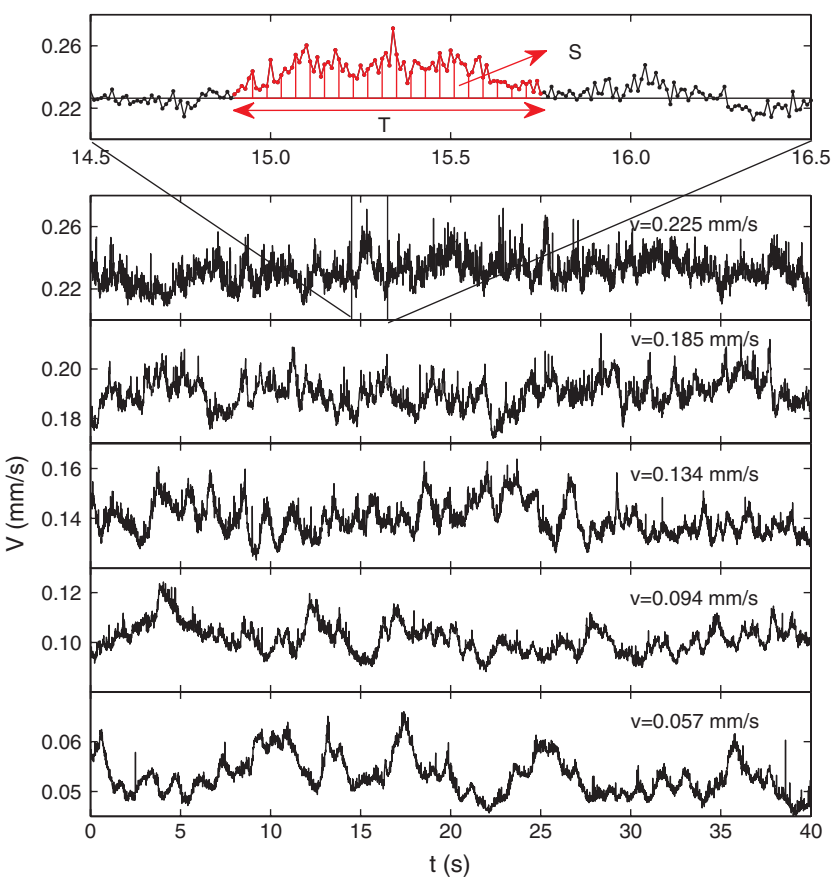

FIG. 1 (color online). Global velocity of the front, $V(t)$, within a window $l=L$ at different rates $v$. The zoomed panel on top reveals the amount of data recorded, and provides the definitions of size $S$ (dashed region) and duration $T$ of an avalanche, for a velocity threshold $V_{c}=\langle V\rangle(c=0)$, represented as the horizontal baseline at $0.225 \mathrm{~mm} / \mathrm{s}$.

level. The disorder patch gives a natural lower cutoff size for the avalanches, $S_{d}=\left(l_{d} / L\right) r=3 \times 10^{-4} \mathrm{~mm}$. We show on the top panel of Fig. 2, in double-logarithmic scale, the size distribution $P(S /\langle S\rangle)$ of the velocity bursts larger than $\langle V\rangle$ (clip level $c=0$ ), for the whole ROI $l=L$ and various injection rates. For each injection rate up to four different realizations of the disorder have been studied. Rescaling the burst size with the average avalanche size $\langle S\rangle$, the data collapse into a single distribution, following clearly a power law with an exponential cutoff $P(X) \propto X^{-\alpha} e^{-X / \xi_{S}}$ with $\alpha \simeq 1.00$ and $\xi_{S} \simeq 4.3$. It is important to mention that this is the first time that such a scaling behavior occurring on a wide range of length scales is observed for the burst dynamics of imbibition fronts. We also analyze for the same conditions the distributions of the burst duration rescaled by the average burst duration $P(T /\langle T\rangle)$. In order to avoid a windowing effect due to the cuttoff size $S_{d}$, and therefore a bias in the distributions at short temporal scales, we considered only avalanches lasting longer than the longest avalanches of size $S_{d}$. Thus, the statistics is reduced and the scaling behavior is more difficult to analyze. However (top inset of Fig. 2), we can still observe clearly that the distributions of the avalanche duration follow as well a power law with an exponential cutoff $P(X) \propto X^{-\tau} e^{-X / \xi_{T}}$, with $\tau \simeq 1.34$ and $\xi_{T} \simeq 3.9$. Both $\langle S\rangle$ and $\langle T\rangle$ increase systematically with $v \rightarrow 0$. The data collapses in Fig. 2 then imply that the distributions $P(S)$ and $P(T)$ approach a pure power law as the true 


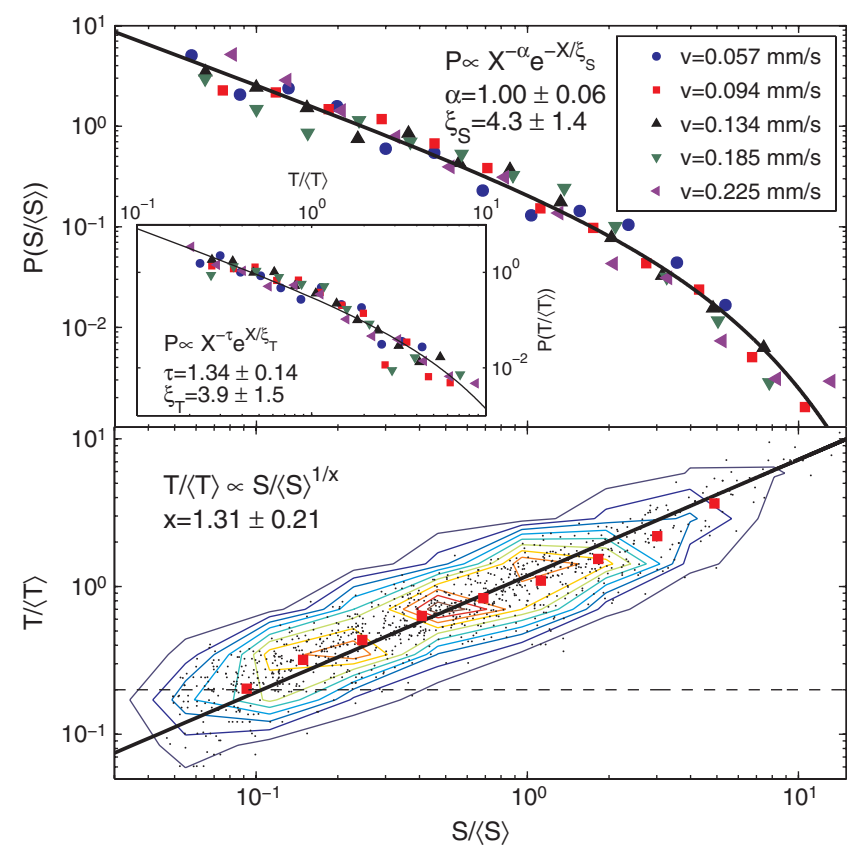

FIG. 2 (color online). Top: Burst size distributions $P(S /\langle S\rangle)$ for the same clip level $c=0$ and various experiments. Inset: Distribution of the avalanche durations $P(T /\langle T\rangle)$ for the same conditions. In both cases the solid line is a power-law fit with an exponential cutoff. Bottom: $(T /\langle T\rangle)$ vs $(S /\langle S\rangle)$ for the same avalanches. Small dots are the values for all experiments and all velocities. Squares are averages over logarithmic bins. The contour levels give the isolines of counts of the joint histogram $P(S /\langle S\rangle, T /\langle T\rangle)$. The solid line is a fit through all the avalanches of the form $S /\langle S\rangle \sim(T /\langle T\rangle)^{x}$ with $x \simeq 1.31$. The dashed line gives the minimum significant duration due to windowing of the avalanche sizes.

critical point $(v=0)$ is approached. Finally, on the bottom panel of Fig. 2, we show the scaling behavior between the duration $T$ and the size $S$ of the avalanches. We observe a power-law behavior $S /\langle S\rangle \sim(T /\langle T\rangle)^{x}$. The scaling exponent has a value $x \simeq 1.31$, which agrees quantitatively with phase-field simulations [10]. We checked that all these scaling behaviors are robust, independent of the injection rate within the range investigated here, the disorder realizations, and for a wide range of clip levels $(-0.5<c<0.75)$.

Fluctuations of $V(t)$.- - Let us turn now to the statistical description of the fluctuations of $V(t)$. The local velocities of the imbibition front at saturation are correlated over a macroscopic length scale. This is visible in Fig. 3, where the statistical width $W_{V}$ of the local velocities is computed in windows of size $l$. For all the injection rates studied, the width grows with $l$ up to a size $l_{\times}$, and remains nearly constant for larger $l$ (uncorrelated region). The correlation length $l_{\times}$can be determined from $W_{V}(l)$ by taking the crossover between the power law and the plateau regions. It decreases with injection rate as $l_{\times} \sim v^{-1 / 2}$, as shown in the inset of Fig. 3 (bottom). It is remarkable that the values of $l_{\times}$actually correspond to the crossover length $\xi_{c}=$ $\sqrt{\kappa / \mathrm{Ca}}$, where $\kappa$ is the permeability of the medium [11].

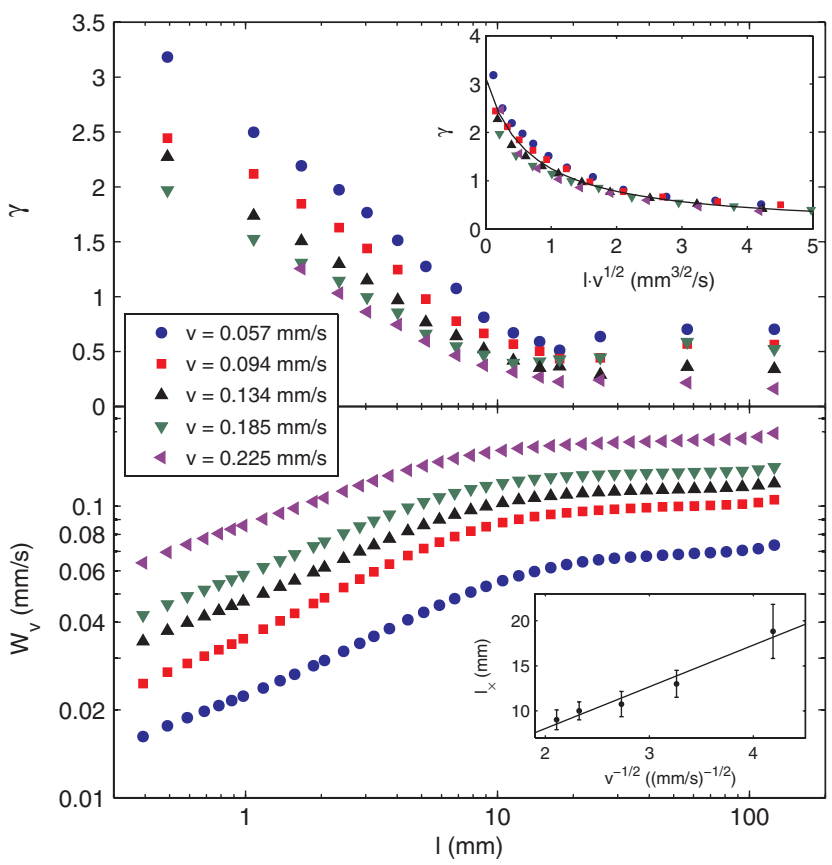

FIG. 3 (color online). Bottom: statistical width of the local velocities as a function of window size $l$, in double-logarithmic scale, for different injection rates. The inset shows that $l_{\times} \sim$ $v^{-1 / 2}$. Top: skewness of the PDF of the fluctuations of $V(t)$ as a function of window size $l$, in semilogarithmic scale. Inset: Data collapse of the skewness as a function of $l v^{1 / 2}$. The solid line is a fit giving $1 / \gamma=0.48\left(l v^{1 / 2}\right)+0.32$.

This crossover length scale, that separates surface tension damping from viscous pressure damping of the interfacial fluctuations [7,13], is the relevant length scale of the problem.

Since the global velocity $V(t)$ shows long-range correlations over large distances, we expect that $V(t)$ will display non-Gaussian fluctuations. Let us consider the normalized variable $Y \equiv(V-\langle V\rangle) / \sigma$. The PDFs of $Y$ are plotted in Fig. 4 for window sizes $l=L$ and $l=$ $L / 40$ and three different injection rates $v$. We find that, at the highest injection rate and over the large window, the fluctuations are nearly Gaussian. However, as the injection rate decreases, the distributions are clearly non-Gaussian: they become progressively more asymmetric, and evidence of an exponential tail for large fluctuations emerges. The effect is much more pronounced for the small window, $l=$ $L / 40 \simeq 3 \mathrm{~mm}$, because this scale of observation is contained within the correlated region for the three injection rates considered here. Results for other window sizes and injection rates, within the range displayed in Fig. 4, follow the same trends. Indeed, we observe in the top panel of Fig. 3 that, for all the various experiments performed, the skewness $\gamma$ (third moment of the distributions) increases systematically when either $l$ or $v$ are reduced. A comparison of the top and bottom panels shows that $\gamma$ does not change appreciably once $l$ becomes larger than the correlation scale $l_{\times}$. 

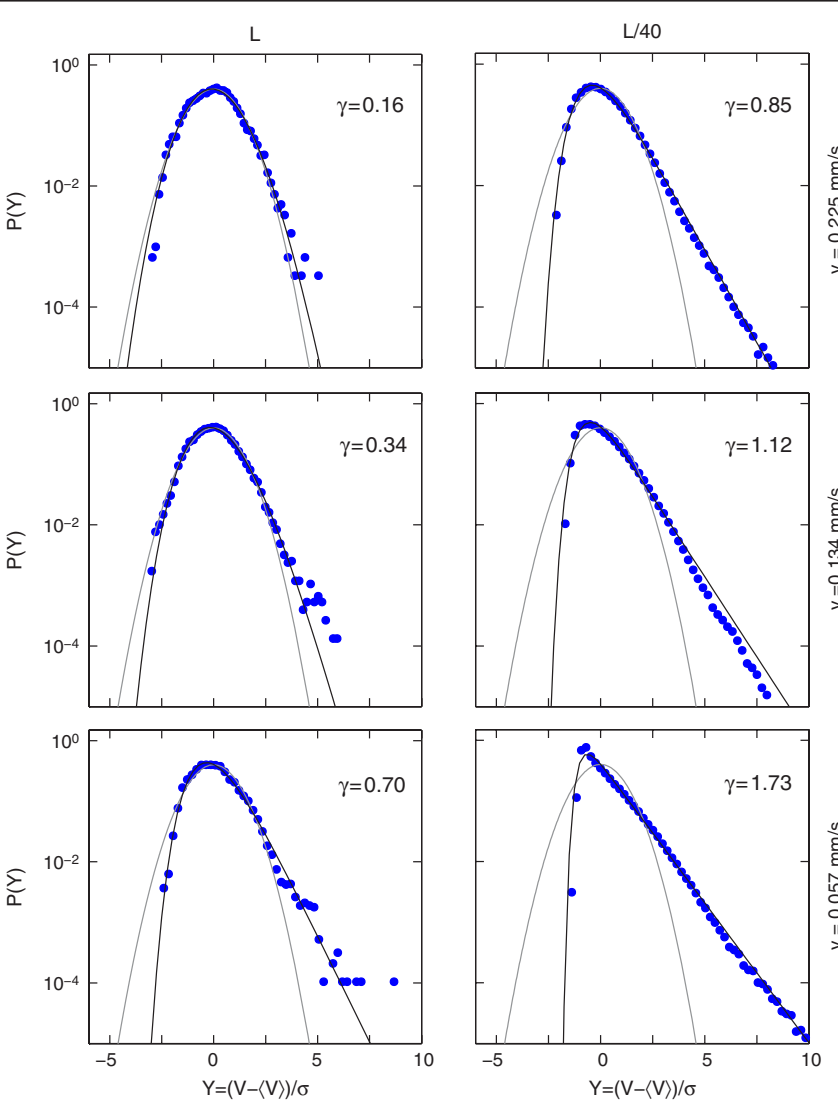

FIG. 4 (color online). PDFs of $(V-\langle V\rangle) / \sigma$ for window sizes $l=L$ and $l=L / 40$ and three different injection rates. The experimental results (dots) include data from one experiment on four different disorder realizations. Thin lines are Gaussian PDFs, and thick lines are GG PDFs obtained using the quoted $\gamma$.

We want now to compare (not to fit) those distributions to a GG distribution $[4,14]$ :

$$
P_{a}(Y)=\frac{a^{a} b_{a}}{\Gamma(a)} \exp \left(-a\left\{b_{a}\left(Y+s_{a}\right)+\exp \left[-b_{a}\left(Y+s_{a}\right)\right]\right\}\right),
$$

where $\quad b_{a}=\sqrt{d^{2} \ln \Gamma(a) / d a^{2}} / \sigma_{Y}, \quad s_{a}=\langle Y\rangle+\{\ln a-$ $[d \ln \Gamma(a) / d a]\} / b_{a}$ and $\Gamma(a)$ is the Gamma function. The only free parameter of $P_{a}(Y)$ is $a$ since $b_{a}$ and $s_{a}$ are fixed by the mean $\langle Y\rangle=0$ and the variance $\sigma_{Y}^{2}=1$ of the global quantity $Y$. The value of the shape parameter $a$ is given by the skewness of the PDF $\gamma \equiv\left\langle Y^{3}\right\rangle \sim 1 / \sqrt{a}[3,4]$. Using the measured skewness for estimating the shape parameter $a$ in Eq. (1), we get the PDFs plotted in Fig. 4 as continuous lines. We find a remarkable agreement with the experimental distributions.

Finally, we compare the spatial extent of the measuring window $l$ with the correlation length $l_{\times}$. The effective number of domains statistically independent can be related to the ratio $N_{\text {eff }}=l / l_{\times}$. Since $l_{\times} \sim v^{-1 / 2}$, we get $N_{\text {eff }} \sim$ $l v^{1 / 2}$. A plot of $\gamma$ as a function of $l v^{1 / 2}$ (top inset in Fig. 3) collapses our data for $\gamma$. Therefore, $\gamma$ provides a measure of the effective number of degrees of freedom probed in our measurements, supporting the theoretical predictions $[3,15]$ that the skewness of the probability distribution of the fluctuations provides an indirect measure of the length scale of correlations in spatially extended correlated systems, as well as recent experimental observations on a second-order equilibrium phase transition [6].

Conclusion.-We have presented an experimental study of the global velocity $V(t)$ of a viscous fluid interface forced to invade a disordered medium at constant flow rate. Our high resolution setup and detailed analysis of the velocity fluctuations has allowed us to characterize with high accuracy the complex intermittent dynamics observed. When the velocity of the front is measured at a scale comparable to its correlation length, the fluctuations of $V(t)$ take a GG shape-an asymmetric non-Gaussian distribution with a large exponential tail—whose skewness gives the effective number of degrees of freedom probed in the experiment. Moreover, we have shown that those large fluctuations - signature of a burstlike dynamics-correspond to avalanches power-law distributed both in sizes and durations. This means that forced-flow imbibition takes place by bursts of the global velocity in a wide range of sizes and durations, with no characteristic scales. Work is in progress in order to characterize the local dynamics of the imbibition fronts and its consequences on the global dynamics investigated here.

We acknowledge useful discussions with K. J. Måløy, J. M. López, M. Palassini, M. Pradas, and A. HernándezMachado. R. P. acknowledges financial support from the DGI (MEC, Spain). This work is supported through MEC, Spain, Project No. FIS2006-03525, and through Generalitat de Catalunya Project No. SGR-2005-00507.

[1] S. T. Bramwell et al., Nature (London) 396, 552 (1998).

[2] S. T. Bramwell et al., Phys. Rev. Lett. 84, 3744 (2000).

[3] B. Portelli et al., Phys. Rev. E 64, 036111 (2001).

[4] E. Bertin, Phys. Rev. Lett. 95, 170601 (2005).

[5] Tibor Tóth-Katona and J. T. Gleeson, Phys. Rev. Lett. 91, 264501 (2003).

[6] S. Joubaud et al., Phys. Rev. Lett. 100, 180601 (2008).

[7] M. Alava et al., Adv. Phys. 53, 83 (2004).

[8] A. Dougherty and N. Carle, Phys. Rev. E 58, 2889 (1998).

[9] A.-L. Barabási and H.E. Stanley, Fractal Concepts in Surface Growth (Cambridge University Press, Cambridge, England, 1995).

[10] M. Rost et al., Phys. Rev. Lett. 98, 054502 (2007).

[11] J. Soriano et al., Phys. Rev. E 66, 031603 (2002).

[12] K. J. Måløy et al., Phys. Rev. Lett. 96, 045501 (2006).

[13] E. Pauné and J. Casademunt, Phys. Rev. Lett. 90, 144504 (2003).

[14] E. J. Gumbel, Statistics of Extremes (Columbia University Press, New York, 1958).

[15] A. Noullez and J.-F. Pinton, Eur. Phys. J. B 28, 231 (2002). 